\title{
Analysis of key aspects to manage Wireless Sensor Networks in Ambient Assisted Living environments
}

\author{
Henar Martín, Ana M. Bernardos, Luca Bergesio, Paula Tarrío \\ Data Processing Group (GPDS CEDITEC) \\ ETSI Telecomunicación - Universidad Politécnica de Madrid \\ Madrid, Spain \\ $\{$ hmartin, abernardos, luca.bergesio, paula\}@grpss.ssr.upm.es
}

\begin{abstract}
Wireless Sensor Networks (WSN) based on ZigBee/IEEE 802.15.4 will be key enablers of non-invasive, highly sensitive infrastructures to support the provision of future ambient assisted living services. This paper addresses the main design concerns and requirements when conceiving Ambient Care Systems (ACS), frameworks to provide remote monitoring, emergency detection, activity logging and personal notifications dispatching services. In particular, the paper describes the design of an ACS built on top of a WSN composed of Crossbow's MICAz devices, external sensors and PDAs enabled with ZigBee technology. The middleware is integrated in an OSGi framework that processes the acquired information to provide ambient services and also enables smart network control. From our experience, we consider that in a future, the combination of ZigBee technology together with a Service Oriented Architecture may be a versatile approach to AAL services offering, both from the technical and business points of view.
\end{abstract}

Keywords- Ambient Assisted Living; context-aware frameworks; OSGi; Wireless Sensor Network; Zigbee

\section{INTRODUCTION}

Ageing societies are looking for sustainable solutions to promote healthy lifestyles and extend independent living for elderly as far as possible. Currently, a remarkable research effort is done in Ambient Care Systems (ACS), architectures designed to provide different types of services both in sensing controlled areas (at home) and outdoors. For example, some of them aim at enhancing the elder's care network [1], detecting and handling emergency situations, helping the target users to accomplish activities of daily living [2] and fulfill medication intake schedules. Remote health monitoring functionalities (even for disease prevention and early diagnosis) and activity pattern recognition [3] are also considered, in particular for patients with Chronic Obstructive Pulmonary Disease (COPD), renal diseases [4], diabetes or (light-mild) Alzheimer [5].

ACS are usually supported by different types of devices and networks, which are supposed to continuously acquire health parameters, ambient information and other context data. In practice, building these networks up imply dealing with different communication technologies. Personal health sensors are usually equipped with Bluetooth, while local area networks are configured on WiFi. Location services -frequently needed for different applications- may be operative on different wireless technologies. Deploying the required infrastructure to make an ACS work is always a time and cost consuming task, which also implies dealing with legacy infrastructure and devices.

In this context, Wireless Sensor Networks (WSN) based on IEEE 802.15.4/ZigBee, devised to non-invasively sense and handle information about users and their environments, become key enablers to make real highly adaptive and personalized care services. Although still in early stage commercial deployment, ZigBee/IEEE 802.15.4 devices and networks offer several benefits to communicate wearable sensors and PDAs or gateways, as they provide more efficient power consumption than other standards such as WLAN or Bluetooth [6]. WSN may be easily deployed and automatically configured in home environments. Moreover, as ACS usually handle sensitive data, it is important to guarantee security issues: in its strongest protection level, ZigBee uses AES 128 encryption and offers a security toolbox for key generation [7]. Additionally, it seems that the more and more devices would be equipped with this technology. For example, in June 2009, Continua Health Alliance has definitively chosen ZigBee as one of the development alternatives of health products [8].

In this paper, the design problems of an Ambient Home Care System (AHCS) relying on a Wireless Sensor Network are addressed. The WSN, would be composed by traditional motes (small and resource-constrained wireless devices with some sensing capabilities) [9], but may include personal mobile devices acting as full-enabled network nodes. From the ACS point of view, by deploying a ZigBee Wireless Heterogeneous Sensor Network at home, it is possible to configure all the functionalities needed to provide AAL services, including location, specific sensor integration and easily deployable solutions.

In our approach, the aforementioned WSN is integrated into an OSGi framework where local inference and decisionmaking tasks are accomplished to provide different services. The main advantages of an OSGi framework rely on its ability to provide resources to develop software that is much less dependent on the hardware and operating system. Apart from that, its Service Oriented Architecture is optimized to easily and remotely manage and create services by defining bundles of code that carry out operations so they can be added together to achieve further functionalities. To our experience, SOA systems are suitable to address the challenges of Ambient Care Systems and their business models. 
The deployment and operation of this OSGi-ZigBee system presents some needs as well as particular problems that should be handled to provide a sound solution. First, it is necessary to equip all the potential nodes in the hybrid sensor network with the ZigBee/IEEE 802.15.4 compatible stack and a shared routing algorithm capable of managing data transmission while preserving power. Secondly, it is important to guarantee that the network may be self-managed, with a minimum of human interaction, and robust to changes in network. Thirdly, location algorithms on ZigBee have to be provided in order to allow that consumer services may use position information. Finally, it is necessary to address the particular integration problems of mobile devices as nodes in the network.

In the following section, the state-of-the-art in platforms for AAL services is described. Section III presents a deeper analysis on the requirements that AAL services impose on WSN-based platforms. Section IV describes the proposed solution, explaining in Section $\mathrm{V}$ how specific integration problems have been addressed. In particular, Section VI explains how the integration of a mobile phone has been done. A summary of open issues concludes the paper.

\section{RELATED RESEARCH}

In the last years, a number of architectures to manage context information have been proposed. Some of them are developed as OSGi frameworks, and a few of them are specifically set to provide services at home.

For example, SOCAM [10] is designed to serve as a general framework to develop context-aware applications. When prepared to provide services in smart environment, the OSGi compliant framework is embedded in a residential gateway which facilitates context acquisition and interpretation. Each SOCAM component (the service-locating service, context interpreters and context providers) is built in a different bundle. A Gateway Operator manages residential gateways and their services.

Another OSGi framework is the one designed by Hein et al. [11] to provide health services at home. Their solution does not specifically address the inclusion of wireless sensor networks, but handle electrocardiogram monitors, digital TV sets, IP telephony and microphones.

The architecture I-Living [13] is not built on OSGi, but based on an Assisted Living Hub (ALH) composed by an operative system with different communication stacks, a middleware providing standard services and, on top of it, the applications code. The ALH acts as the local intelligence and manages peripherals through its own peripheral network, usually based on Bluetooth. A special feature of I-Living is that it makes emphasis in protecting data over-the-air and information confidentiality.

Kang et al. [1] describe a middleware whose aim is to make easier the interoperability between health sensor manufacturers and health service providers. Their middleware is based on an OSGi framework that is supported by a context model built on an ontology developed with OWL. The system gathers the information from two health ZigBee devices: a clock (with an ECG sensor, a photoplethysmograph sensor and a skin temperature sensor) and a chest belt (with an ECG sensor, a respiration sensor, a SKT sensor and a three axis acceleration sensor).

The integration of ZigBee technology in health sensors has also been addressed in [14], where Dağtaş et al. explain the use of ZigBee sensors to acquire ECG data. Huang et al. [15] specifically describe the implementation of a heart rate monitor (Panasonic EW 3035) with ZigBee connectivity.

With respect to hardware alternatives to configure a ZigBee/IEEE 802.15.4 infrastructure, different commercial products are available. Table 1 briefly compares the main characteristics of the most popular platforms for WSN.

TABLE I. COMPARISON OF SENSOR NETWORK PLATFORMS

\begin{tabular}{|c|c|c|c|c|}
\hline \multirow[b]{2}{*}{ Platforms } & \multicolumn{4}{|c|}{ Features } \\
\hline & Standard & Sensor inclusion & $\begin{array}{c}\text { Programming } \\
\text { language }\end{array}$ & $\begin{array}{l}\text { Power } \\
\text { source }\end{array}$ \\
\hline MICAz & $\begin{array}{l}\text { IEEE } \\
802.15 .4 \\
\text { compliant }\end{array}$ & $\begin{array}{l}\text { Sensor boards } \\
\text { provided with } \\
\text { analog and } \\
\text { digital outputs. }\end{array}$ & nesC & $\begin{array}{c}\text { Batteries } \\
(3 \mathrm{~V})\end{array}$ \\
\hline SunSPOT & $\begin{array}{l}\text { IEEE } \\
802.15 .4\end{array}$ & $\begin{array}{l}\text { Temperature, } \\
\text { light, humidity } \\
\text { and analog and } \\
\text { digital outputs. }\end{array}$ & Java & $\begin{array}{l}\text { Lithium } \\
\text { battery }\end{array}$ \\
\hline Squidbee & $\begin{array}{l}\text { IEEE } \\
802.15 .4 \text { or } \\
\text { ZigBee }\end{array}$ & $\begin{array}{l}\text { Temperature, } \\
\text { light, humidity } \\
\text { and possibility } \\
\text { to add new } \\
\text { ones. }\end{array}$ & Arduino & $\begin{array}{c}\text { Battery } \\
(9 \mathrm{~V})\end{array}$ \\
\hline ANT & $\begin{array}{l}\text { ANT } \\
\text { Protocol }\end{array}$ & $\begin{array}{l}\text { Temperature, } \\
\text { digital inputs. }\end{array}$ & $\begin{array}{c}\text { scrips } \\
\text { SensRcore } \\
\text { en NVM }\end{array}$ & $\begin{array}{l}\text { Coin cell } \\
\text { batteries }\end{array}$ \\
\hline $\begin{array}{l}\text { Sentilla } \\
\text { JCREATE }\end{array}$ & $\begin{array}{l}\text { ZigBee } \\
(802.15 .4)\end{array}$ & $\begin{array}{l}\text { Accelerometers, } \\
2 \text { standard } \\
\text { connectors and } \\
\text { an expansion } \\
\text { connector. }\end{array}$ & Java & $\begin{array}{l}2 \text { AAA } \\
\text { batteries }\end{array}$ \\
\hline
\end{tabular}

In previous literature, WSN integration in AAL frameworks is usually not described, but treated as a 'black box'. It is expected that our research will complement preceding works by exposing the problems and solutions needed to integrate ZigBee/IEEE 802.15.4 heterogeneous WSN (including external devices equipped with ZigBee technology) in an OSGi architecture in order to provide AAL services.

\section{REQUIREMENTS FOR WSN IN AMBIENT ASSISTED LIVING ENVIRONMENTS}

In this Section, the AAL service scenario which motivates this contribution is described. Following there is a list of the functional requirements which need to be addressed in order to have a reliable system based on WSN.

\section{A. Ambient Care Systems: Service Scenario.}

The design objectives motivating this work are related to the development of an Ambient Care System capable of offering its services at home and outdoors. The ACS will be composed by:

a. A Personal Network (PN), which will include all the devices that a person must wear or use to allow the services to work. The elderly's personal network will include a ZigBee device (a ZigBee tag or a PDA equipped 
with ZigBee technology) to enable indoor positioning, while the user is supposed to take his PDA with him when outdoors. Additionally, depending on the user profile and the services to be configured, the PN may include continuous monitoring sensors (ECG, oxymeters, etc.) and other health sensors (e.g. wireless spirometers). PDAs or smartphones may act as light processing units and gateways to centralized systems when needed, and they may be ready to interact with augmented objects through ZigBee interfaces. To configure the PN, it is always important to bear in mind the usability restrictions imposed by the user's acceptance of personal devices in home environments.

b. A Home Network, which will include home infrastructure sensors (ambient, presence, pressure, home automation sensors, etc.), actuators and appliances capable of notifying their status. The Home Network Unit will be able to communicate with the Personal Network by using ad hoc networking capabilities. It will include local intelligent features to dispatch events and orders depending on the situation. These processing capabilities will be part of a home gateway which will connect the home environment with the Core Care Network.

c. A Core Care Network, serving as a bridge of communication between the home infrastructure and third parties and service providers. Services may be enabled through the Core Care Network. It can also authorize the connection of external service providers, centralize system monitoring and guarantee the security of personal data.

On top of this structure, general services such as: a) context-aware notification dispatching, b) emergency detection, c) activity logging or d) health monitoring are to be configured. The first service allows the user or the caregiver to subscribe to events such as medical dates or biomedical and environmental information notification. The second one relies on the processing of biomedical data related to an illness or environmental parameters that can worsen the illness. In this way an action can be taken as soon as the emergency is detected. Thanks to service c) all the information about activity or events that has been produced is stored to allow an analysis of the evolution of the environment. Finally, d) makes possible to establish whether the user must be always monitored or has to put on a specific sensor in a specific moment to get an important measurement. It is also possible to program the frequency of the measurements of the sensors. Moreover, for specific users, such as COPD patients, additional features related to their disease will be included. This will require aggregating new sensing technologies and different processing and data fusion algorithms.

\section{B. General design requirements for WSN in Ambient Care} Systems.

The previous scenario imposes some functional requirements to the Wireless Sensor Network finally composed by infrastructure and personal sensing nodes. Next there is a brief list of the most important features to consider:

- Interoperability. Wireless Sensor Networks in real deployments need to be ready to manage heterogeneous sensors, which need to share a common communication scheme. Some of these sensors may be embedded in mobile devices, so it is important that interoperability standards are supported both for autonomous nodes and PDAs or smartphones. Although different technologies (such as Bluetooth or WiFi) may be currently suitable to support interoperability due to their integration in mobile devices, ZigBee seems to be a better option for the future. To make ZigBee available in different types of devices, it is necessary to have embeddable ZigBee stacks.

- Network self-configuration and maintenance. It is desirable that the WSN demands as little attention from a human operator as possible. The nodes of the network should be always aware of the configuration of the network, knowing which nodes are their neighbors and the information they can get from them, adapting to events. To achieve this goal, the network should be able to manage the inclusion or removal of new sensors and to control the batteries level of the nodes.

- Easy and robust deployment. When designing WSN functionalities, it is important to consider the deployment requirements to make the network fully operational. For example, commercial products or services will impose that the WSN may be deployed by following a non-supervised process, which minimizes calibration tasks and optimizes the number of infrastructure nodes to be added. But nodes cannot be randomly deployed. Their positions must be chosen, for example, to allow their sensors to monitor the ambient characteristics in points where these measurements provide relevant information about the environment. Moreover, it is important to take into consideration the propagation of the signal and, in this way, avoid to place the nodes at positions where the signal is expected to be severely affected by multipath or fading (e.g. surrounded by many objects). This precaution will prevent the deployment of unnecessary sensors acting as mere forwarders and will facilitate the location estimation. As a consequence, it is important to count on deployment methodologies which may adapt to the particularities of the environment and services.

- Multihop routing. A WSN for Ambient Care Systems usually consists of several sensor nodes that send their measurements to a sink node, which collects all the information and typically sends it to a PC, where all the data are stored and elaborated. In a home, the sink node may not provide coverage over the whole area. As a consequence, it is necessary to implement routing algorithms that transmit the information towards the sink through other nodes. There are many proposals of different routing algorithms for sensor networks (e.g. LEACH (Low Energy Adaptive Clustering Hierarchy), SPIN (Sensor Protocols for Information via Negotiation), Minimum Cost Forwarding Algorithm (MCFA), etc. [16]). The routing protocol should be able to manage the network dynamically, being able to change the routing paths when the topology of the network is modified due to the death of a node or the appearance of a new node, for example. As these routing algorithms may need to work in a mobile device, it is important to evaluate their quality and 
resources consumption not to overload the PDA or smartphone.

- Positioning service. This kind of service is required for many operational and service purposes. For instance, to process the information related to the place where the user is, avoiding the storage and computation of information that is not relevant in a specific moment. It has also a principal role in the inference of activity or behavior patterns, which can result in firing alarms if the person has stayed in the same place for longer than usual or if it seems that he is wandering. In the case of applications for inclusion it can also help to guide people with disabilities to their destiny. Due to technology usability and acceptance reasons, it is useful to implement location capabilities by using different type of location devices. For ACSs, tags or mobile phones will be considered.

- Energy saving strategies. As the devices that are integrated in the network have limited computational and radio communication capabilities, collaborative algorithms with energy-aware communication are required to achieve multi-modal collaboration and energy conservation. A typical strategy to achieve this purpose is, for example, to allow the node to switch to sleep mode periodically, wake up to receive information and process it and go back to sleep when this is finished. ZigBee technology is supporting the implementation of low-energy location and routing protocols, so this is one of the main reasons to choose this technology for ACSs.

- Scalability of sensors and actuators. ACS's services may impose different type of sensing and actuation requirements. For example, in a scenario considering services for COPD patients, devices sensing the quality of air may be needed. For that reason, WSN for ACSs need to be ready to include new sensors and actuators, which may be connected to existent network nodes or configure as nodes themselves. Methodologies and software architectures making easier to scale the network sensing capabilities are needed.

- Integration of mobile phones as WSN nodes. ZigBee technology could be integrated in personal mobile devices in the near future, including them as processing nodes. Nowadays these devices can be integrated by using external cards, but in the future they will be integrated directly into the SIM card of each mobile phone. ZigBee protocol is based on IEEE 802.15.4, so this kind of devices can take part to a wireless sensor network using IEEE 802.15.4. By including mobile devices as nodes, location information together with acceleration data, light sensors or cameras images may be acquired and processed to infer complex information.

- Connecting the WSN to the Internet. Providing sensor nodes with an IP address makes possible their integration as web services. In 2007, the rfc4944 - Transmission of IPv6 Packets over IEEE 802.15.4 Networks - was published. This RFC defines an adaptation of IPv6 for wireless sensor networks called 6LoWPAN. Nowadays ZigBee is more widespread than 6LoWPAN, and hardware availability of sensors and network devices that support IPv6 is limited, but with this technology it is possible to communicate directly with sensors through Internet and to use well known routing algorithms and services using the same network stack. It exists also a hybrid solution called IP over ZigBee that uses IPv6 addresses over ZigBee network layer and maintains ZigBee for higher layers.

- $\quad$ Security. As wireless networks are based on a standard and data are sent over a broadcast channel, it is possible to make packet sniffing and data spoofing attacks. IEEE 802.15.4 MAC layer offers some facilities which can be used by upper layers to achieve a good level of security. The standard implements the Rijndael algorithm (AES); it uses a specific shared key, provided by higher-layer processes, to perform symmetric cryptography and to protect the payload. Moreover the standard uses an Access Control List (ACL) to register MAC addresses of nodes and to permit or deny communications. With these two features and using some additional packet fields, the IEEE 802.15.4 guarantees access control, message integrity, confidentiality and reply protection. Anyway, the security level of the entire system is determined by higher layers. ZigBee specification implements a basic security model using IEEE 802.15.4 security features with 128-bit shared keys so it is possible to implement a secure system handling only higher layers.

\section{AN OSGI FRAMEWORK TO MANAGE WIRELESS SENSOR NETWORKS}

Figure 1 shows the architecture of the proposed framework, which configures the Home Network Unit. It consists of a Home Network Infrastructure and an OSGi middleware hosting acquisition and reasoning features; both of them are described below.

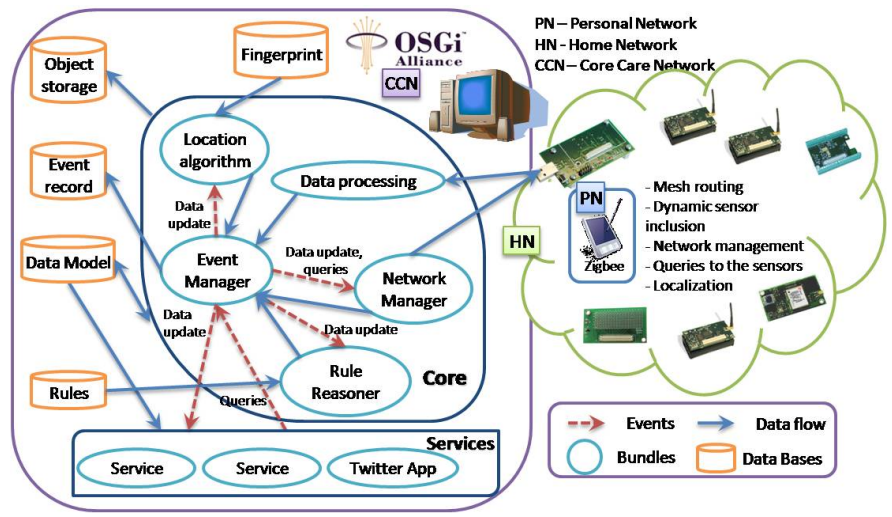

Figure 1. Framework architecture.

\section{A. The Home Network infrastructure.}

The sensor network performing acquisition tasks at home is composed of IEEE 802.15.4/ZigBee wireless modules, and PDAs or mobile phones equipped with a miniSD card to enable them with ZigBee technology. In particular, the sensor network that has been integrated into the framework is composed of MICAz nodes from Crossbow. These modules are IEEE 802.15.4 compliant, so it has been necessary to add a special stack (see Section VI) to enable ZigBee communications 
among them and the mobile phones that are also part of the network.

The infrastructure nodes have attached some sensor boards (MDA100, MDA300 and MTS420, distributed by Crossbow) to monitor light, humidity, temperature as well as accelerometers and GPS estimation of location (outdoors). Additionally, external sensors have been attached for different purposes; e.g. pressure mats have been connected to support activity inference and location services, while quality of air sensors have been integrated for the monitoring scenario for COPD patients.

The information provided by the sensors is routed with a multihop algorithm to the base station (or sink node), which is connected to a PC that hosts the Home Network Unit. The information consumers are the services developed inside the middleware, but external applications might access context and user data too. The information sent by the wireless modules is sent to the modules through a subscription mechanism.

\section{B. The Home Network Unit on an OSGi Middleware.}

The middleware handling the Wireless Sensor Network has been implemented into an OSGi framework (Figure 2). OSGi provides a complete and dynamic component model to run over Java. The applications or components are deployed in the framework as bundles (jars files with extra manifest headers) and can be remotely managed, that is, they can be installed, started, stopped, updated and uninstalled without requiring a reboot. The Service Layer connects bundles in a dynamic way. The Life Cycle Management controls the status of the bundle. The purpose of the Service Registry layer is to detect the addition of new services, and the removal of existing services, and allows the framework to adapt accordingly. The Modules Layer defines how a bundle can export and import code and finally, the Security Layer controls that the bundle is deployed securely. OSGi suits our AAL framework functional needs as it decouples data acquisition, data processing, multipurpose algorithms and services, so these functions can be easily shared among all the services, making easier code reuse.

The AAL OSGi framework runs in a computer that, additionally, works as a gateway to receive the information from the sensor network and transmit it to the bundles. Consumer services can be developed based on the information provided by the bundles of the core part of the architecture. The OSGi framework databases store the information shared in the network.

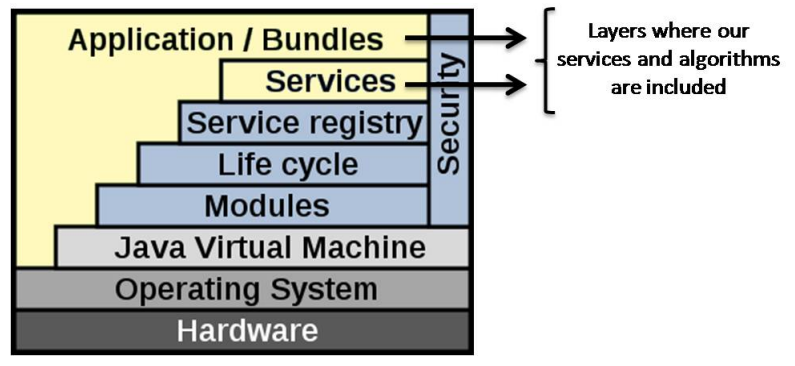

Figure 2. Generic OSGi architecture, with the layers in which our services and algorithms have been implemented.
Our middleware runs on the Eclipse Equinox (www.eclipse.org/equinox/) implementation of the OSGi specifications, which is certified to fulfill R4 by the OSGi Alliance [17].

The framework works on a data model, which is needed to share information efficiently. The data and event model serves for all the services to agree on the same objects and the same attributes to describe every element of the network and the relationships among them.

\section{IMPLEMENTATION OF WSN REQUIREMENTS IN AN AAL OSGI FRAMEWORK.}

To fulfill some of the requirements described in Section III, different solutions have been adopted in the OSGi framework presented in Section IV. Following there is a brief description on how these technical challenges have been addressed.

\section{1) Providing location estimates.}

For most part of AAL services, location is an important context feature which may be an input for reasoning engines. Therefore, a localization algorithm providing symbolic (zone) localization of the MICAz motes as well as the ZigBee enabled PDAs has been included in the framework. The localization algorithm uses the received signal strength to estimate the target position. In practice, the RSS is obtained from the field strength of the TOS Msg packet defined in Crossbow's software platform developed under TinyOS-1.x. Our algorithm relies in the known position of some motes of the network, hereafter known as beacon nodes. Fingerprinting is used to estimate the position [18] as follows: first of all, a calibration phase is carried out to measure and store the RSS values measured from the different beacon nodes in different points of the area (RSS map). Next, in real time (the online stage), the RSS from the mobile node (PDA or mote) is measured at all the beacon nodes in the area of coverage. These measurements are compared to the ones stored in the RSS map: the points of the map with a closer fingerprint are selected and their positions are averaged. The node is finally assumed to be located in the room that contains the point resulting from the previous step. Another possibility to compute the location is using an algorithm based on a channel model. A channel model is a function that establishes a relation between the distance between two nodes and the RSS measured. Once we estimate the distance from the mobile node to at least three beacon nodes, the position is computed using a triangulation algorithm. The room where the node is placed is assumed to be the one of the estimated position.

\section{2) Mesh routing.}

The environment of deployment of this framework makes necessary the implementation of a routing algorithm since, as there is only one sink in the sensor network, it will not provide coverage over the whole area. In order to include this functionality in the network the application Surge (provided by the TinyOS-1.x distribution) is modified to cover our needs. This application uses the libraries from the directory lib/Route to use the implementation of a router named MultihopRouter. This implementation uses a shortest-path algorithm with a single destination node (the root) and active two-way link estimation based on packet loss statistics. Every node stores a table that contains the information of the father and the 
neighbors, including the hop counts and the link quality estimation. These tables are broadcasted to build the routes. When a packet is being transmitted, the next hop destination is decided based on shortest path semantics. This is equivalent to building a tree with the sink as the root. As it is mentioned before, packet loss statistics are also taken into consideration for deciding the next hop destination. They are estimated by listening to every received packet (including snooping, that is listening to packets addressed to other nodes), and analyzing their sequence numbers. In case that the next packet received is out of order, it is concluded that some packets were lost. However, since links may be asymmetric (good in one direction, but poor quality in the other) the quality is estimated for both ways. In figure 4 the structure of the components of this application is presented [9] [19].

In our case a new packet structure to spread the information through the whole network is defined. The code has been adapted to measure the values from different sensor boards using Crossbow drivers. In this way, the components of the application related to the micasb sensor board changes depending on the sensor board that is used.

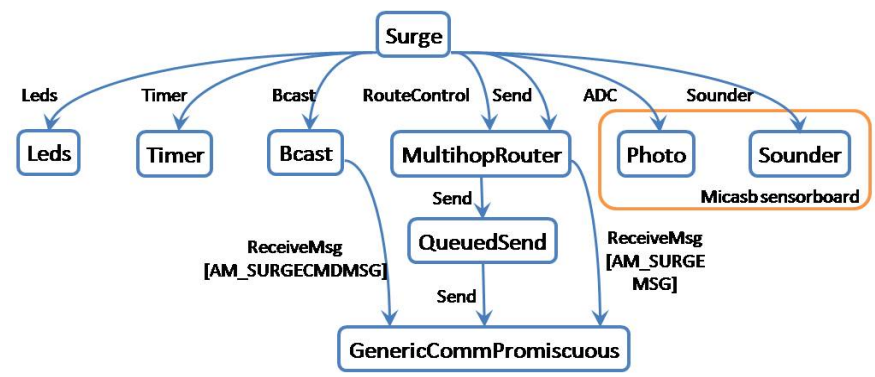

Figure 3. Structure of the components of the Surge application where the part that is modified in our application is framed.

\section{3) Querying the sensors}

The possibility of providing sensor measurements under demand, as well as periodically, enables many new AAL services that would not exist if the communication was only unidirectional (from sensors to Home Network Unit). For this reason, this functionality has been implemented in the proposed OSGi framework. When the OSGi framework running in the $\mathrm{PC}$ receives a request of a measurement or measurements, it must forward a query to the sensor network to carry out the measurement.

In order to do this TinyOS provides some Java Tools to offer robust and flexible serial communication. The querying functionality has been implemented using the MIG (Message Interface Generator) tool to automatically generate Java classes that correspond to the Active Message types of TinyOS. MIG reads in the nesC structures definitions for those message types and generates a Java class for each message type that takes care of packing and unpacking fields of the message.

The package net.tinyos.message is also used, as it contains a number of routines for sending and receiving messages through the MIG-generated message classes. The NCG (nesC Constant Generator) tool is also used to extract constants from nesC files [19].

\section{4) Network management}

The management of the network includes features such as adding new sensors to the network, updating information about some sensors, replacing batteries in case that a mote has run out of power, etc. When a new node or sensor is introduced in the network, two different approaches (static and dynamic) can be used to manage this change. The static approach consists in stopping the framework, including the new motes or sensors and updating the information about existing devices in the configuration file that is processed when the framework is launched. The cost of stopping and resuming the framework can be assumed when the WSN is monitoring a home or living space, as the number of nodes or sensors and their characteristics are not expected to change frequently, mainly once the trial period of the framework has finished. In the dynamic approach, a field of the packet structure of the sensors is used to indicate whether new information is introduced in the framework. The motes send the packets with this flag activated during the first minute after the introduction of new features to try to guarantee that the packets reach the sink node; the framework continuously monitors this field and, when it detects the activated flag, it sends an acknowledgment to notify that the information has been added correctly.

In case that a mote runs out of batteries an alarm is sent to the services subscribed to this type of events. As mentioned, the framework monitors the packets that are received from the network and they have a field with the battery of the node. A timer controls the reception time of the last packet from each node; if this time exceeds three minutes and the battery level of the last packet was low the event 'out of batteries' for that specific node is sent. In case the battery level was normal an event 'node failure' is sent.

\section{5) Adding external sensors}

Not all the sensors that are necessary to monitor the ambient are included in the sensor boards available for the MICAz motes. Regarding the problem of attaching a new sensor (e.g. a pressure mat, and air quality sensors), the acquisition board MDA300 from Crossbow has been used. Using the digital I/O and ADC channels included in this board, readings from external sensors can be easily obtained and stored [9].

In the case of the pressure mat, its output signal was connected to one of the available digital channels. Therefore, when the sensed value is 0 , an event updating that the person is over the mat is sent. Air quality sensors demand different ways to be attached to the sensor boards. For example, it may be necessary to attach them to one of the precise differential ADC channels. Other sensors may need extra circuitry if they require power supply or if their output signal is higher than the input voltage range of the channel. In this last case we must implement a voltage divider.

\section{INTEGRATING THE MOBILE PHONES AS NODES OF A WSN}

Mobile phones and PDAs need to be part of AAL platforms. In our approach, PDAs are integrated into the WSN as network nodes. This enables location services and direct communication to other nodes. 
Nowadays mobile phones and PDAs are not yet equipped with ZigBee technology, and the integration of external technology (SD cards) derives in certain problems related to interoperability. The wireless sensor network that is used in our AAL system is based on IEEE 802.15.4 standard, so it is necessary to give mobile phones and PDAs the ability to communicate with that standard. A ZigBee compliant radio system integrated within a SD card is used to communicate with the network. The first two layers of ZigBee follow the IEEE 802.15.4 standard, but the network layer and its routing protocol are parts of ZigBee specifications and it cannot be neither changed nor deleted on commercial SD cards. Our sensors have IEEE 802.15.4 compliant hardware and they use TinyOS. TinyOS offers some modules to handle the radio transceiver and the MAC layer, but it does not have a network layer module to exchange messages with ZigBee devices. For that reason, a network module has been developed in TinyOS to make possible the communication between ZigBee devices and sensors based on TinyOS.

Figure 4 shows a ZigBee frame, without MAC layer data and divided into network, application support sublayer and application data. That is a typical frame which has been necessary to analyze in order to develop the interoperability module.

\begin{tabular}{|c|c|c|}
\hline NWK data & APS data & APP data \\
\hline $4800000001000 A 07$ & 400006800000 & $000404000100010 B$ \\
\hline
\end{tabular}

Figure 4. ZigBee frame without MAC layer. MAC data is composed of source and destination address, source and destination PAN address, sequence number, CRC16 and packet length. NWK data has a control field ( 2 bytes), a network destination address (2), a network source address (2), a broadcast radius (1) and a broadcast sequence number (1). The application support sublayer (APS) data is composed of a control field (1), a destination endpoint (1), a cluster ID (1), a profile ID (2) and a source endpoint (1).

As it is not possible controlling any SD card parameters (for example source address, PAN address, profile, etc.), all mobile phones and PDAs are configured as end points (Reduced Function Device) so they cannot route packets. They can only receive data from sensors and send packets for localization purposes. With this TinyOS module, mobile phones and PDAs can be integrated into the network and the device-assisted localization service described in Section $\mathrm{V}$ can be implemented.

\section{CONCLUSIONS AND FURTHER WORK}

In this paper, the functional requirements to be considered when designing an AAL framework working on a ZigBee/IEEE 802.15.4 network are addressed. Our proposal uses an OSGi architecture to cope with sensor integration and provide general services on WSN which are scalable both in ZigBee sensor integration and processing capabilities.

From the point of view of new sensors, we are envisaging the integration of health monitor devices equipped with ZigBee technology. Our goal is to make these devices to interoperate directly with the infrastructure sensor network when the elderly is at home, for him not to carry a gateway device (PDA).

Enhancing communication between different types of ZigBee stacks and IEEE 802.15.4 is also a key point to make easier the integration of different types of devices and sensors. For example, developing a full compliant ZigBee 2006-2007 stack for Crossbow motes is an open issue.

With respect to reasoning services, activity inference algorithms and behavioral pattern analysis are to be included in our OSGi framework. These features may be especially useful for applications focused on mentally challenged people.

Security is another aspect to improve. For example, a new layer on the communication stack to implement a security system based on shared keys is needed. Apart from that, it is important to consider the design of strategies to guarantee authentication, integrity, confidentiality and availability when getting/providing information from/to external partners. This aspect can be managed with a hierarchical system of permissions and standard tools of web security.

Another line of research is distributed computation and control to prevent the bottlenecks and single-point failures that may appear in the presented approach, as all the information has to be sent to the sink node to be processed by the OSGi framework.

In our ACS, ZigBee has been chosen as sensing technology because the number of ZigBee-compliant devices is increasing, while the majority of wireless sensors use IEEE 802.15.4. Additionally, it provides security features at high level which are very important for the type of services relying on an ACS. Nevertheless, it is necessary to keep an eye on other emerging technologies (e.g. IP over ZigBee, EtherBee2, RIPlink, 6LoWPAN, ROLL, etc.) which are designed to integrate the Internet Protocol (IP) in order to permit communications with objects, nodes and sensors using existent network infrastructure.

\section{ACKNOWLEDGMENT}

This work has been supported by the Government of Madrid under grant S-0505/TIC-0255 and by the Spanish Ministry of Science and Innovation under grant TIN200806742-C02-01. Henar Martín and Paula Tarrío acknowledge the Spanish Ministry of Education for their grants. The authors also acknowledge related discussions with partners within the AmIVital Cenit project, financed by the Centre for the Development of Industrial Technology (CDTI).

\section{REFERENCES}

[1] S. Consolvo, P. Roessler, B.E. Shelton, A. LaMarca, B. Schilit, S. Bly, "Technology for Care Networks of Elders", IEEE Pervasive Computing, vol. 3, no. 2, pp. 22-29, 2004.

[2] M.E. Pollack, L. Brown, D. Colbry, C.E. McCarthy, C. Orosz, B. Peintner, S. Ramakrishnan, I. Tsamardinos, "Autominder: An Intelligent Cognitive Orthotic System for People with Memory Impairment", Robotics and Autonomous Systems, vol. 44, no. 3, pp. 273-282 (10), 2003.

[3] D.J. Cook, "Health Monitoring and Assistance to Support Ageing in Place", Journal of Universal Computer Science, vol. 12, no. 1, 2006."

[4] R. Farré, A. Papadopoulos, G. Munaro, R. Rosso, "An Open, Ubiquitous and Adaptive Chronic Disease Management Platform for Chronic Respiratory and Renal Diseases", Int. Conf. on eHealth, Telemedicine and Social Medicine, pp. 184-188, 2009.

[5] S. Zhang, S. McClean, B. Scotney, X. Hong, C. Nugent, M. Mulvenna, "Decision Support for Alzheimer's patients in Smart Homes", $21^{\text {st }}$ IEEE Int. Symposium on Computer-Based Medical Systems, pp. 236-241, 2008. 
[6] M. Kohvakka, M. Kuorilehto, M. Hännikäinen, T.D. Hämäläinen, "Performance Analysis of IEEE 802.15.4 and ZigBee for Large-Scale Wireless Sensor Network Applications", ACM Int. Workshop on performance evaluation of wireless ad hoc, sensor and ubiquitous networks, pp. 48 - 57, 2006.

[7] N. Sastry, D. Wagner. "Security Considerations for IEEE 802.15.4 Networks". Proceedings of the 2004 ACM Workshop on Wireless Security.

[8] Continua Alliance Press Release, June 8, 2009: http://www.continuaalliance.org/static/cms_workspace/Continua_06082 009_vFINAL.pdf

[9] Wireless sensor Networks - Getting Started Guide, Crossbow, 2005, pp. 36-47.

[10] T. Gu, H.K. Pung, D.Q. Zhang, "Towards an OSGi-Based Infrastructure for Context-Aware Applications", Pervasive Computing, pp. 66-74, 2004.

[11] A. Hein, M. Eichelberg, O. Nee, A. Schulz, A. Helmer, M. Lipprandt, "A Service Oriented Platform for Health Services and Ambient Assisted Living", Int. Conf. on Advanced Information Networking, pp. 531-537, 2009.

[12] D. Kang, K. Kang, H. Lee, E. Ko, J. Lee, “A Systematic Design Tool of Context Aware System for Ubiquitous Healthcare Service in a Smart Home," in Future generation communication and networking, vol. 2, pp.: 49-54, Dec. 2007.
[13] Q. Wang, W. Shin, X. Liu, Z. Zeng, C. Oh, B.K. AlShebli, M. Caccamo, C.A. Gunter, E. Gunter, J. Hou, , K. Karahalios, L. Sha, "I-Living: An Open System Architecture for Assisted Living", IEEE International Conference on Systems, Man and Cybernetics (SMC '06), Vol.: 5, pp. 4268-4275, 2006.

[14] S. Dağtaş, G. Pekhteryev, Z. Sahinoglu, "Multi-stage Real Time Health Monitoring via ZigBee in Smart Homes", IEEE Int. Conf. on Advanced Information Networking and Applications Workshops, pp. 782-786, 2007.

[15] M-C. Huang, J-C. Huang, J-C. You, G-J. Jong, "The Wireless Sensor Network for Home-Care System Using ZigBee", Int. Conf. on Information Hiding and Multimedia Signal Processing, vol. 1, pp. 643646, 2007.

[16] J.N. Al-Karaki and A.E. Kamal, "Routing techniques in wireless sensor networks: a survey," in Wireless Communications, vol. 11, no. 6, pp. 628, 2004.

[17] OSGi Alliance: http://www.osgi.org/Main/HomePage.

[18] H. Martín, P. Tarrío, A. M. Bernardos, and J. R. Casar, "Experimental Evaluation of Channel Modelling and Fingerprinting Localization Techniques for Sensor Networks", Proc. $2^{\text {nd }}$ International Workshop on User-Centric Technologies and Applications (MADRINET'08), pp. 3241, 2008.

[19] TinyOS Tutorials: http://www.tinyos.net/tinyos-1.x/doc/index.html. 